\title{
Adaptive Fuzzy Integral Sliding Mode Control Application to the Launch Vehicle's Orbital Injection Problem
}

\author{
U. Ansari and A. H. Bajodah \\ Aeronautical Engineering Department, Faculty of Engineering, King \\ Abdulaziz University, P.O. Box 80204, Jeddah 21589, Saudi Arabia
}

\begin{abstract}
This paper presents an application of adaptive fuzzy integral sliding mode control to the longitudinal attitude tracking problem of satellite launch vehicles. A direct adaptive Fuzzy control law is set to be the equivalent part in the sliding mode control expression. This equivalent part strives to imitate a perfect feedback linearization of the vehicle's longitudinal attitude dynamics by approximating the unknown functions in the vehicle's model. The singleton control action of the fuzzy control law is set to be an adjustable parameter, and the adaptive mechanism is augmented to tune this parameter with respect to the changing environment in order to reduce the residual error between fuzzy control and feedback linearization control. The adaptation mechanism is designed based on Lyapunov principle, and it guarantees asymptotic stability of the closed loop system. The reaching (switching) part of the sliding mode control law attempts to constrain the system's state to remain on the sliding surface. An external guidance loop is designed to reshape the reference attitude profile online in order to attain desired orbital altitude and injection angle. To analyze the controlled ascent flight trajectory, a nonlinear 3 Degrees of Freedom numerical simulation model is developed for a four stage Launch Vehicle, and performance evaluation of the proposed control system is conducted on the model in the presence of model parametric uncertainties and external disturbances.
\end{abstract}

Keywords: Launch vehicle attitude control; Adaptive fuzzy integral sliding mode control; Lyapunov stability principle; Launch vehicle guidance; Real time estimation.

\section{Introduction}

Fuzzy control has been an active topic of research for the last few decades. It explicitly makes use of human's experience and knowledge about the system, and it is specifically suitable for systems that are ill modeled with uncertain and complex dynamics. A fuzzy control 
algorithm is considered to be a nonmathematical control algorithm. It is made up of heuristic decision rules, which differentiates it from conventional feedback control algorithms, see, e.g. ${ }^{[1,2]}$.

The number of heuristic rules in a fuzzy control algorithm grows exponentially with the number of inputs to the fuzzy control generator ${ }^{[3]}$. One way to overcome this curse of dimensionality is to augment fuzzy control principles with sliding mode control principles $[4,5]$. The advantage of this methodology is not limited to reducing the number of fuzzy rules, but it also achieves a better closed loop performance as compared to many individual control methodologies ${ }^{[6]}$.

The sliding surface in a sliding mode control algorithm is a function of tracking error and its rate, and the control objective is to drive this surface to zero. Moreover, since uncertainties, nonlinearities, and external disturbances acting on the system are not well known, the dynamics of the system cannot be precisely modeled. To overcome this problem, fuzzy control plays the role of approximating unknown system functions in the sliding surface expression ${ }^{[7]}$.

Similar to conventional adaptive control, there are two approaches to adaptive fuzzy control: direct and indirect. In the direct approach, the adaptive mechanism tunes the controller parameters to reduce the system's performance error variables ${ }^{[8]}$, whereas in the indirect approach ${ }^{[9]}$, the adaptive law is responsible for identifying the unknown plant parameters. In both approaches, fuzzy rules are automatically adjusted to attain the desired closed loop performance, see, e.g., ${ }^{[7,10-12]}$.

In Ref. ${ }^{[13]}$, a direct adaptive fuzzy sliding mode control (AFSMC) is applied to a linear model of the longitudinal dynamics of a satellite launch vehicle (SLV). In continuation of our research work on SLV guidance and control ${ }^{[14-16]}$, a direct AFSMC design that is similar to the one introduced in ${ }^{[17]}$ is presented in this paper to control the SLV's longitudinal nonlinear attitude dynamics such that the SLV achieves the desired orbital parameters. The integral sliding surface is based on the pitch attitude error and its rate. The employed adaptive control element is based on Lyapunov principle, and it aims to automatically adjust the singleton fuzzy control action.

To achieve desired orbital altitude and injection angle, an external guidance loop is used for online reshaping of the pitch attitude profile that is to be tracked by the SLV. The paper is organized as follows. In section 2, a 3 Degrees of Freedom (DOF) mathematical model of a SLV 
is presented. Section 3 describes the design procedure of the direct AFSMC methodology, and the reference attitude trajectory guidance algorithm is briefly explained in section 4. Simulation results and conclusion are presented in section 5 and 6 , respectively.

\section{3DOF Simulation Model of Satellite Launch Vehicle}

The data of a four-stage SLV has been used to generate the reference attitude trajectory ${ }^{[18]}$, and it is used in this paper to design the control law and to evaluate its performance. Table 1 depicts the fundamental parameters of the four-stage SLV.

Table 1. Data of satellite launch vehicle.

\begin{tabular}{lllll}
\hline \hline Parameters & Stage-1 & $\begin{array}{l}\text { Stage- } \\
2\end{array}$ & $\begin{array}{l}\text { Stage- } \\
2\end{array}$ & Stage-4 \\
\hline Stage mass (ton) & 31.34 & 9.31 & 2.87 & 0.91 \\
Mass of Propellant (ton) & 18.76 & 5.55 & 1.72 & 0.54 \\
Thrust (kN) & 593 & 262 & 94 & 30 \\
Mass flow rate (kg/s) & 265 & 103 & 37 & 13 \\
Stage Burning time (s) & 70.7 & 53.8 & 46.9 & 46.9 \\
Diameter of Stage (m) & 1.3 & 1.0 & 0.7 & 0.5 \\
\hline \hline
\end{tabular}

By using the Launch Vehicle data, a 3DOF simulation model is developed in MATLAB/SIMULINK $®$ having the following approximate longitudinal dynamical model by assuming rigid body dynamics and 1976 standard atmosphere ${ }^{[19]}$.

$$
\begin{aligned}
\left(\begin{array}{c}
\dot{V}_{x l} \\
\dot{V}_{y l} \\
\dot{V}_{z l}
\end{array}\right)=\frac{1}{m} L_{l b}\left(\begin{array}{c}
-Q S C_{D} \\
Q S C_{l \alpha} \alpha+0.5 T \sin \delta_{\theta} \\
0
\end{array}\right)+L_{l e}\left(\begin{array}{c}
g_{x e} \\
g_{y e} \\
g_{z e}
\end{array}\right)-L_{l e}\left[\left(\begin{array}{c}
0 \\
0 \\
\omega_{E}
\end{array}\right)^{\mathrm{x}}\left(\begin{array}{c}
0 \\
0 \\
\omega_{E}
\end{array}\right)^{\mathrm{x}}\left(\begin{array}{c}
x_{e} \\
y_{e} \\
z_{e}
\end{array}\right)\right]-L_{l e}\left[2\left[\begin{array}{c}
0 \\
0 \\
\omega_{E}
\end{array}\right)^{\mathrm{x}}\left(\begin{array}{c}
V_{x e} \\
V_{y e} \\
V_{z e}
\end{array}\right)\right] \\
J_{z} \dot{\omega}_{z}-\left(J_{x}-J_{y}\right) \omega_{y} \omega_{x}=-L\left(x_{c p}-x_{c g}\right)-\frac{T\left(x_{r}-x_{c g}\right) \delta_{\theta}}{2} \\
\theta=\varphi+\alpha .
\end{aligned}
$$

Where $\left(\begin{array}{c}0 \\ 0 \\ \omega_{E}\end{array}\right)^{\mathrm{X}}=\left[\begin{array}{ccc}0 & -\omega_{E} & 0 \\ \omega_{E} & 0 & 0 \\ 0 & 0 & 0\end{array}\right]$.

In the above equations, $\theta$ is Euler pitch angle, $\alpha$ is the angle of attack, $\varphi$ is the flight path angle, $T$ is the total thrust force, $L$ is the lift force, $D$ is the drag force, $Q$ is the dynamic pressure, $\omega_{E}$ is earth's rotational speed relative to the inertial frame, $\omega_{x}, \omega_{y}, \omega_{z}$ are angular body rates, $S$ is the body cross sectional area, $C_{D}$ is the drag coefficient, $C_{l \alpha}$ is 
the lift coefficient derivative with respect to $\alpha, \delta_{\theta}$ is the control deflection in the pitch channel, $L_{l e}$ and $L_{l b}$ are the transformation matrices from earth to launch and from body to launch frames, respectively ${ }^{[19]}$. The vectors $X_{e}=\left[\begin{array}{lll}x_{e} & y_{e} z_{e}\end{array}\right]^{T}, V_{e}=\left[\begin{array}{lll}V_{x e} & V_{y e} V_{z e}\end{array}\right]^{T}, \quad$ and $g_{e}=\left[g_{x e} g_{y e} g_{z e}\right]^{T}$ are the position, the velocity, and the acceleration due-to-gravity vectors respectively, all expressed in the earth frame, whereas $V_{l}=\left[V_{x l} V_{y l} V_{z l}\right]^{T}$ is the velocity vector, expressed in the launch frame. The axes of the body frame in which the equations of motion were derived are chosen to be the principal body axes of the SLV, and the corresponding principal moments of inertia are $J_{x}$ and $J_{y}$, respectively. The axial lengths from the SLV's nose to its center of mass and center of pressure are $x_{c g}$ and $x_{c p}$, respectively, and $x_{r}$ is the axial distance from the SLV's nose to the motor hinge line, see Fig. 1. In Eq. (2), $x_{c p}-x_{c g}$ and $x_{r}-x_{c g}$ are the aerodynamic moment arm and the control moment arm, respectively.

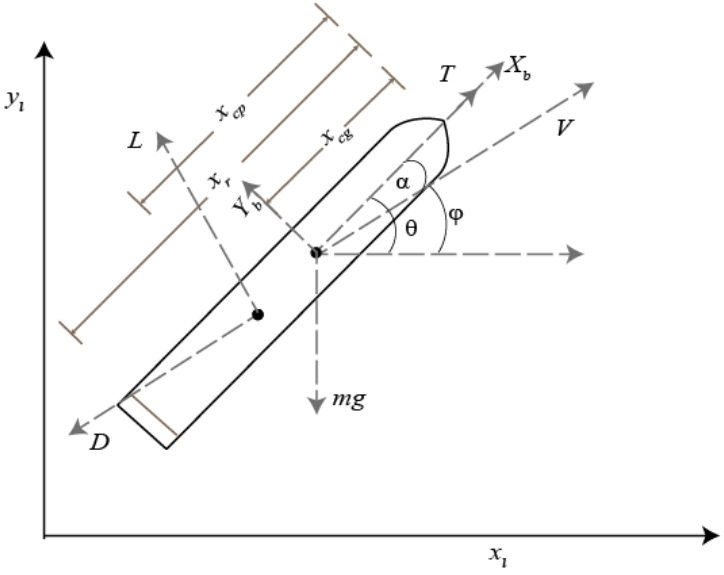

Fig. 1. Planar motion of vehicle.

The desired orbital parameters of the four-stage SLV are given in Table $2^{[15]}$.

Table 2. Orbital Parameters.

\begin{tabular}{ll}
\hline \hline Injection orbit & Desired values \\
\hline Altitude $(\mathrm{km})$ & 500.0 \\
Injection Velocity $(\mathrm{m} / \mathrm{s})$ & 7612.60 \\
Angle of Injection $(\mathrm{deg})$ & 0 \\
\hline \hline
\end{tabular}


Subsequent sections describe the detailed design process of the AFSMC control and guidance systems that aim to inject the Satellite in the desired orbit.

\section{Design of Adaptive Fuzzy Integral Sliding Mode Autopilot}

The linguistic information in fuzzy control that is incorporated for decision making has been proven effective in controlling many practical systems and acquiring improved control performance. However, the fuzzy rule base of a conventional fuzzy system for a dynamical system that contains $p$ input variables has $R^{p}$ rules, where $R$ is the number of fuzzy sets in a single membership function. The size of the rule base therefore grows exponentially with the number of input variables of the control system ${ }^{[3]}$. A remedy for that problem is to define a sliding surface as a function of the error and its rate. As a consequence, the number of rules reduces significantly in fuzzy sliding mode control (FSMC) as compared to conventional fuzzy control. A nonlinear secondorder single-affine input system is given by

$$
\ddot{\theta}=f(\theta, \dot{\theta}, t)+g u
$$

If the system function $f(\theta, \dot{\theta}, t)$ and the constant input matrix $g$ are exactly known a priori, then a perfect feedback linearization control law is given by

$$
u^{*}=\frac{1}{g}\left[-f(\theta, \dot{\theta}, t)+\ddot{\theta}_{c}(t)-k_{1} \dot{e}(t)-k_{2} e(t)\right]
$$

where $e(t)=\theta-\theta_{c}(t), k_{1}$ and $k_{2}$ are prescribed positive gains, and $\theta_{c}(t)$ is the reference pitch attitude. In reality, it is impossible to determine the exact mathematical model of the system due to the presence of strong nonlinearities and uncertainties. Therefore, it is preferable to augment the baseline feedback linearization control law with an adaptive component to automatically adjust the control parameters such that the feedback linearization approximation of the closed loop system is enhanced and closed loop stability is guaranteed.

In the proposed methodology, the input to the system is an integral sliding surface ${ }^{[20]}$ as depicted in Fig. 2, which is selected as the function of error and the error rate as defined below

$$
s(t)=\dot{\theta}(t)-\int_{0}^{t}\left[\ddot{\theta}_{c}(\tau)-k_{1} \dot{e}(\tau)-k_{2} e(\tau)\right] d \tau .
$$




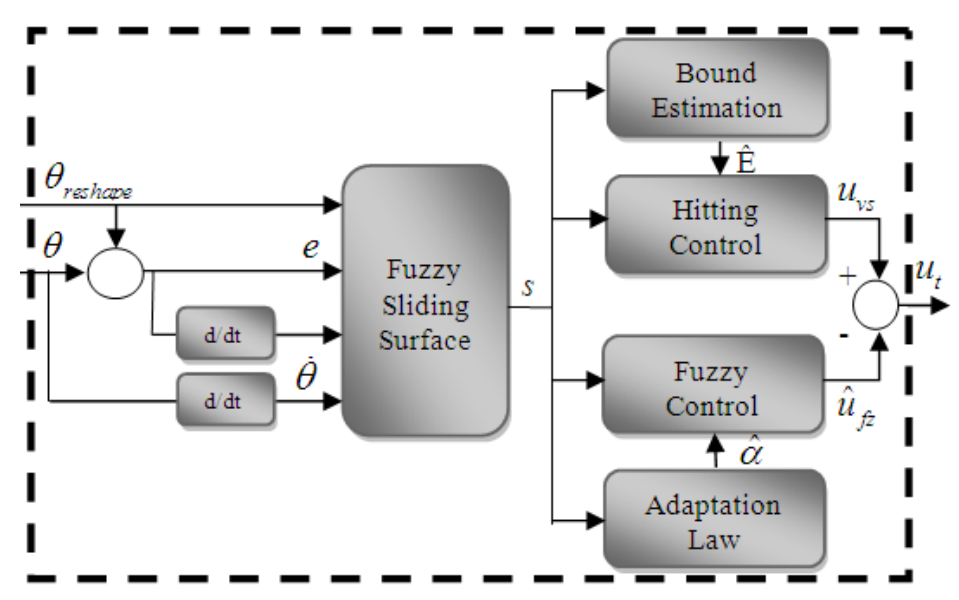

Fig. 2. Adaptive Fuzzy Integral Sliding Mode Control ${ }^{[24]}$.

In ideal state, the sliding surface is $s(t)=\dot{s}(t)=0$, which yields

$$
\dot{s}=\ddot{e}(t)+k_{1} \dot{e}(t)+k_{2} e(t)=0 .
$$

In fuzzy control, the triangular shaped membership functions [21] are used to make the IF part for each input variable. For each individual rule, the subsequent part is the singleton control action $\alpha_{i}, i=1,2, \ldots, n$ depicted in Fig. 3, where $n$ represents the number of fuzzy rules. The singleton fuzzy control action $a_{i}$ is selected to be a tunable parameter that adjusts itself with respect to the surrounding environment ${ }^{[22]}$.

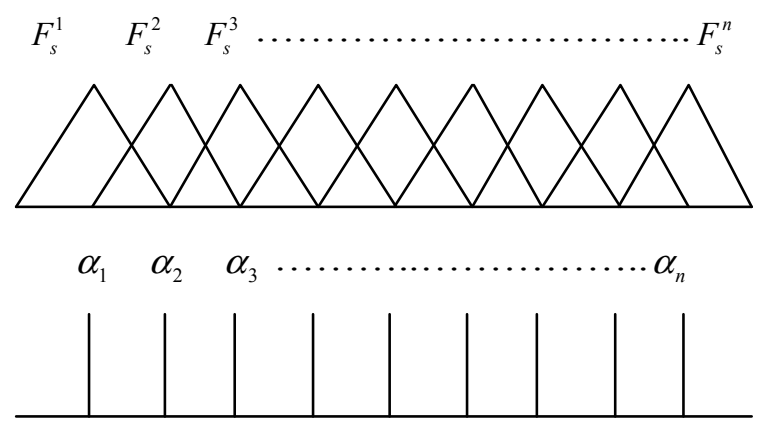

Fig. 3. Singleton control action.

The $i_{t h}$ fuzzy rule in the design process is written as:

Rule $i$ : IF $s$ is in the domain of $F_{s}^{i}$, THEN $u_{f_{c_{i}}}$ is $\alpha_{i}$.

The defuzzification of the control output is accomplished by the center of gravity method, as shown below ${ }^{[15]}$. 


$$
u_{f c}(\alpha, \xi)=\frac{\bigsqcup_{i=1}^{m} w_{i} \cdot a_{i}}{\bigsqcup_{i=1}^{m} w_{i}}
$$

where $w_{i}$ is the firing weight of the $i_{t h}$ rule. The adaptive FSMC law can be explicitly expressed as a function of integral sliding surface and adjustable parameter.

$$
u_{f c}(\alpha, \xi)=a^{T} \xi
$$

where $\alpha=\left[\alpha_{1}, \alpha_{2}, \ldots, \alpha_{m}\right]^{T}$ and $\xi=\left[\xi_{1}, \xi_{2}, \ldots, \xi_{m}\right]^{T}$ represent the parameter vector and regressive vector respectively with $\xi_{i}$ defined as

$$
\xi_{i}=\frac{w_{i}}{\sum_{i=1}^{m} w_{i}} .
$$

Based on the universal approximation theorem ${ }^{[23]}$, the perfect feedback linearization control can be expressed as

$$
u^{*}=u_{f c}^{*}\left(\alpha^{*}, \xi\right)+\varepsilon=\alpha^{*} \xi+\varepsilon .
$$

The approximation error $\varepsilon$ is described as

$$
\varepsilon=u^{*}-u_{f c}^{*}\left(\alpha^{*}, \xi\right)
$$

The perfect feedback linearization control $u^{*}$ can be approximated by the estimation of fuzzy control law $u_{f c}^{*}\left(\alpha^{*}, \xi\right)$ as

$$
\hat{u}_{f c}(\hat{\alpha}, \xi)=\hat{\alpha}^{T} \xi
$$

where $\hat{\alpha}$ is the estimation of $\alpha^{*}$. By incorporating this, the designed Adaptive FSMC is now listed as

$$
u=\hat{u}_{f c}(\hat{\alpha}, \xi)+u_{v s} \text {. }
$$

In Eq. (14), $\hat{u}_{f c}$ is the fuzzy based control law which is designed to approach $u^{*}$, whereas $u_{v s}$ is the switching law in order to minimize the error between the designed fuzzy law $\hat{u}_{f c}$ and perfect feedback linearization control law $u^{*}$.The estimated error between the adaptive fuzzy law and the perfect feedback linearization control yields 
$\tilde{u}_{f c}=\hat{u}_{f c}-u^{*}=\hat{u}_{f c}-u_{f c}^{*}-\varepsilon$.

The tunable parameter can be estimated as

$\tilde{\alpha}=\hat{\alpha}-\alpha^{*}$.

By solving Eqs. (5) and (7) we obtain

$$
\begin{aligned}
& u^{*}=\frac{1}{g}[g u-\dot{s}(t)] \\
& \dot{s}(t)=g\left[\hat{u}_{f z}+u_{v s}-u^{*}\right] .
\end{aligned}
$$

The adaptive control element is designed by employing a Lyapunov function ${ }^{[24]}$ as shown below

$$
V_{1}(s(t), \tilde{\alpha})=\frac{1}{2} s^{2}(t)+\frac{g}{2 \gamma_{1}} \tilde{\alpha}^{T} \tilde{\alpha}
$$

where $\gamma_{1}$ is constant. The time derivative of the Lyapunov function is

$$
\begin{aligned}
\dot{V}_{1}(s(t), \tilde{\alpha}) & =s(t) \dot{s}(t)+\frac{g}{\gamma_{1}} \tilde{\alpha}^{T} \dot{\tilde{\alpha}}=s(t) g\left(\hat{u}_{f z}+u_{v s}-u^{*}\right)+\frac{g}{\gamma_{1}} \tilde{\alpha}^{T} \dot{\tilde{\alpha}} \\
& =s(t) g\left(\tilde{\alpha}^{T} \xi+u_{v s}-\varepsilon\right)+\frac{g}{\gamma_{1}} \tilde{\alpha}^{T} \dot{\tilde{\alpha}}=g \tilde{\alpha}^{T}\left(s(t) \xi+\frac{1}{\gamma_{1}} \dot{\tilde{\alpha}}\right)+s(t) g\left(u_{v s}-\varepsilon\right) .
\end{aligned}
$$

To ensure the asymptotic stability of the system i.e. $\dot{V}<0$, the adaptive law is now written as

$$
\begin{aligned}
\dot{\tilde{\alpha}}=\dot{\hat{\alpha}} & =-\gamma_{1} s(t) \xi \\
u_{v s} & =-E \operatorname{sign}(s(t)) .
\end{aligned}
$$

Now Eq. (20) is written as

$$
\dot{V}_{1}(s(t), \tilde{\alpha})=-E|s(t)| g-\varepsilon s(t) g \leq-E|s(t)| g+|\varepsilon||s(t)| g=-(E-|\varepsilon|)|s(t)| g \leq 0 .
$$

Negative semi-definiteness of $\dot{V}_{1}(s(t), \tilde{\alpha})$ implies Lyapunov stability of the system equilibrium $(s(t), \tilde{\alpha})=(0,0)$. Asymptotic stability follows from Barbalat lemma, see, e.g., ${ }^{[25]}$. The next objective in the design process is to find the reaching phase gain $E$. If $E$ is chosen too small then this causes inaccuracy in staying over the sliding surface, and if $E$ is too large then there are chances that the system may become unstable because of chattering around the sliding surface. Hence, it would be preferable to design $E$ adaptively in order to gain tracking accuracy and 
avoid closed loop instability. By replacing $E$ by $\hat{E}$, the equation of the hitting (reaching phase) control law is written as

$$
\hat{u}_{v s}=-\hat{E}(t) \operatorname{sign}(s(t))
$$

Where $\hat{E}$ is the estimated bound of the approximation error. The estimation error is defined as

$$
\tilde{E}(t)=\hat{E}(t)-E \text {. }
$$

A Lyapunov function $V_{2}$ is now defined to force the states such as $s(t)$ and $\tilde{\alpha}$ tends to zero. The function $V_{2}$ is written as

$$
V_{2}(s(t), \tilde{\alpha}, \tilde{E})=\frac{1}{2} s^{2}(t)+\frac{g}{2 \gamma_{1}} \tilde{\alpha}^{T} \tilde{\alpha}+\frac{g}{2 \gamma_{2}} \tilde{E}^{2} .
$$

Differentiating with respect to time yields

$$
\begin{aligned}
\dot{V}_{2}(s(t), \tilde{\alpha}, \tilde{E}) & =s(t) \dot{s}(t)+\frac{g}{\gamma_{1}} \tilde{\alpha}^{T} \dot{\tilde{\alpha}}+\frac{g}{\gamma_{2}} \tilde{E} \dot{\tilde{E}}=g \tilde{\alpha}^{T}\left(s(t) \xi+\frac{1}{\gamma_{1}} \dot{\hat{\alpha}}\right)+s(t) g\left(u_{v s}-\varepsilon\right)+\frac{g}{\gamma_{2}} \tilde{E} \dot{\tilde{E}} \\
& =-\hat{E}(t)|s(t)| g-\varepsilon s(t) g+\frac{g}{\gamma_{2}}[\hat{E}(t)-E] \dot{\hat{E}}(t) .
\end{aligned}
$$

In order to attain $\dot{V}_{2} \leq 0$, the estimation law is defined as

$$
\dot{\hat{E}}(t)=\gamma_{2}|s(t)| \text {. }
$$

By putting the value of estimation error, Eq. (27) can be written as

$$
\begin{aligned}
\dot{V}_{2}(s(t), \tilde{\alpha}, \tilde{E}) & =-\hat{E}|s(t)| g-\varepsilon s(t) g+[\hat{E}-E]|s(t)| g \\
& =-\varepsilon s(t) g-E|s(t)| g \leq|\varepsilon||s(t)| g-E|s(t)| g=-(E-|\varepsilon|)|s(t)| g \leq 0 .
\end{aligned}
$$

Negative semi-definiteness of $\dot{V}_{2}(s(t), \tilde{\alpha}, \tilde{E})$ implies Lyapunov stability of the system equilibrium $(s(t), \tilde{\alpha}, \tilde{E})=(0,0,0)$. Asymptotic stability follows from Barbalat lemma, see, e.g., ${ }^{[25]}$.

\section{Guidance Mechanism}

In the design process, external guidance law will generate the reshaped reference pitching profile which is accurately followed by inner loop Adaptive FSMC Autopilot. In launch vehicles, guidance mechanism gives steering commands and directs the vehicle to achieve desired injection parameters within the specific bound of accuracy and without compromising vehicle's structural loading limit. A simplest guidance 
mechanism is developed in this study to keep the vehicle on its nominal track; this nominal trajectory is pre-computed on the basis of mission objectives. The differences $\Delta x, \Delta y$ are computed between current range $x_{l}(t)$ and required range $x_{\text {lref }}(t)$ and current altitude $y_{l}(t)$ with desired altitude $y_{\text {lref }}(t)$. From the differences $\Delta x, \Delta y$, a correction angle $\sigma$ is computed online as shown in Fig. 4, this angle reshapes the nominal pitch angle profile. This online reshaping brings the vehicle back to its desired path even in perturbed flight conditions.

$$
\begin{aligned}
& \Delta x=x_{l}(t)-x_{\text {lref }}(t) \\
& \Delta y=y_{l}(t)-y_{\text {lref }}(t) \\
& \sigma=\tan ^{-1}\left(\frac{\Delta y}{\Delta x}\right) \\
& \theta_{\text {reshape }}=\theta_{c}+\sigma .
\end{aligned}
$$

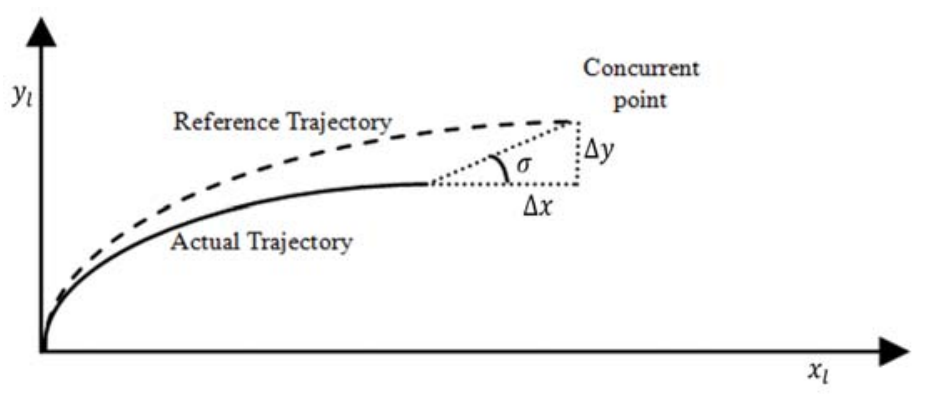

Fig. 4. Guidance mechanism.

An engine shutoff mechanism is implemented during the third stage to achieve required orbital velocity for circular orbit. The criterion for engine shutoff depends on the magnitude of semi major axis $(a)$ given by Eq. (33), which is calculated online and compared with reference values, by the end of third stage, when it achieves reference value; thrust from the engine turn to zero.

$$
a=\frac{|r| \mu}{2 \mu-r v^{2}} .
$$

Where $\mu=G M$ in which $G$ is Gravitational constant and $M$ is Mass of Earth. The vectors $r$ and $v$ are position and velocity vectors defined as $r=\sqrt{x_{l}^{2}+\left(y_{l}+R_{e}\right)^{2}+z_{l}^{2}} \& v=\sqrt{V_{x l}^{2}+V_{y l}^{2}+V_{z l}^{2}}$. The block diagram of the overall system is depicted in Fig. 5. 


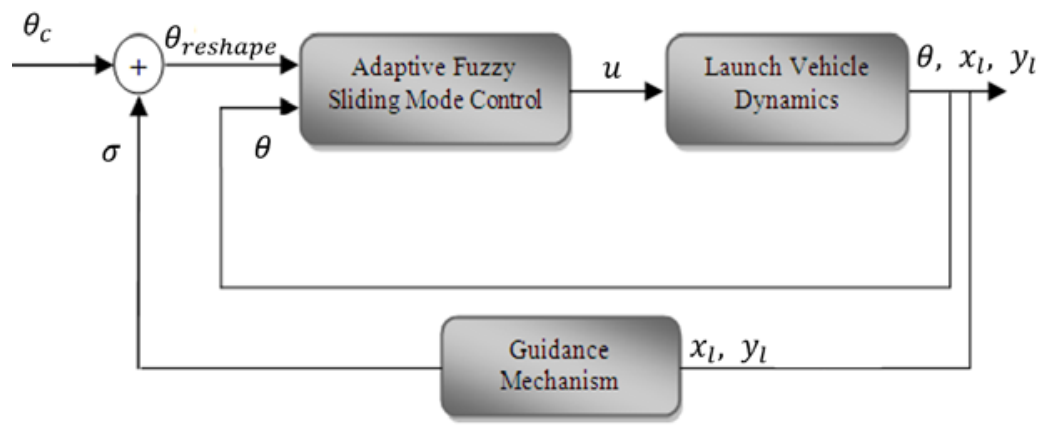

Fig. 5. Adaptive fuzzy based pitch autopilot.

\section{Simulation Results}

At first, the performance of AFSMC is enumerated through 3 DOF simulation model with nominal flight conditions wherein disturbances in atmospheric and mass properties are not included. Figures 6 and 7 present the followed pitch angle with its reference and the control command generated by AFSMC respectively for initial pitch over flight phase.

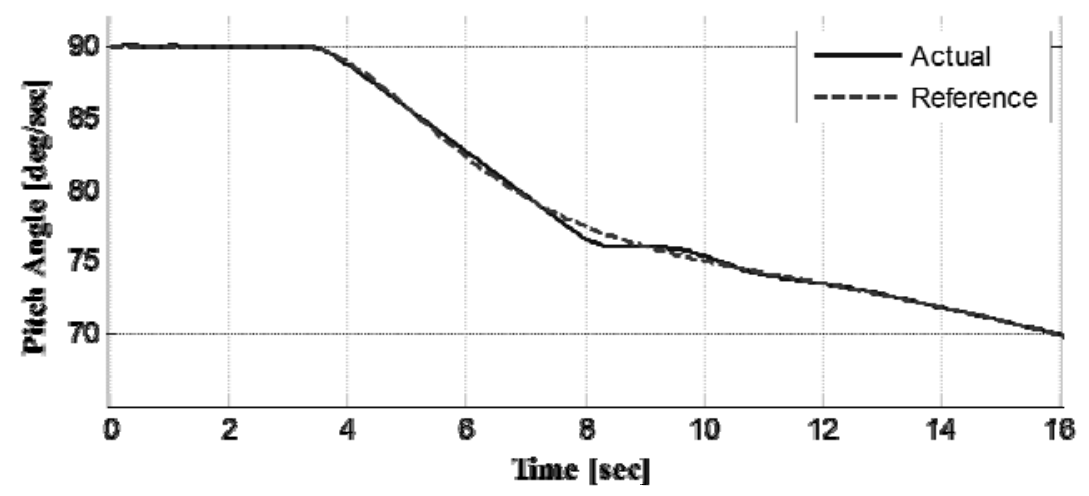

Fig. 6. Pitch control in initial pitch over.

For initial pitch over phase, above figures portray the effectiveness of proposed control scheme by precisely steering the system on the reference pitch angle. Figures 8 and 9 give the complete flight profile and deflections from launch to injection.

Based on above nominal conditions, a perturbed flight conditions are established, to check the robust performance of AFSMC, in which aerodynamic coefficients variation and thrust variation are included. Table 3 gives the quantification of applied disturbances whereas all these 
disturbances are applied in such a way that if applied in together gives either altitude increasing or decreasing as show in Fig. 10.

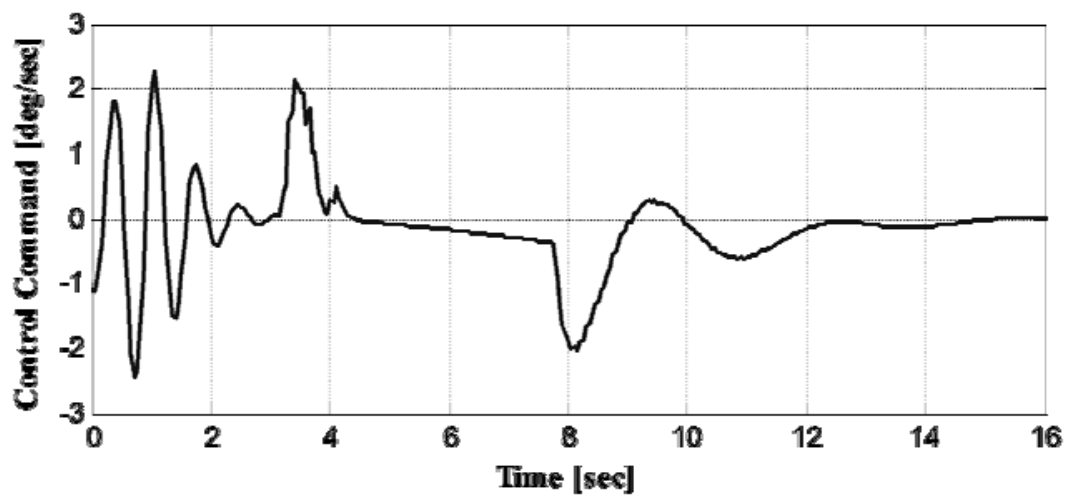

Fig. 7. Control deflection in initial pitch over.

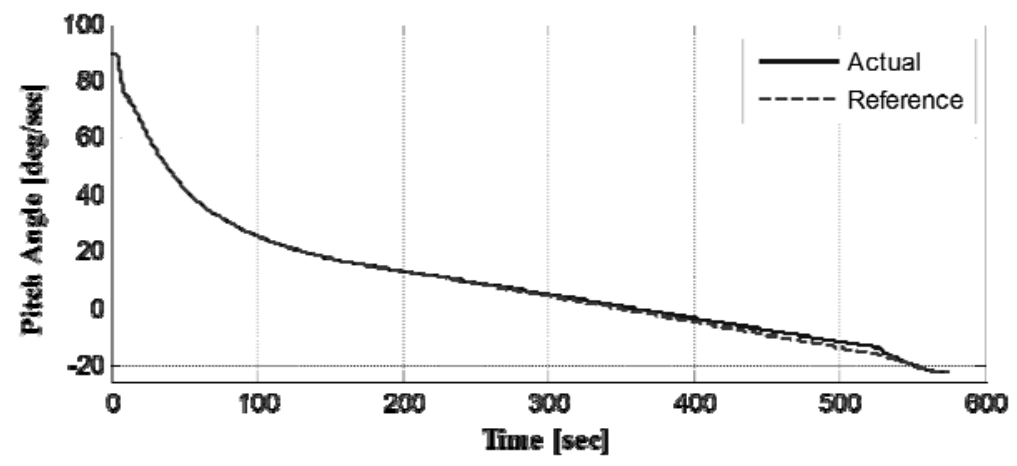

Fig. 8. Pitch angle vs. time.

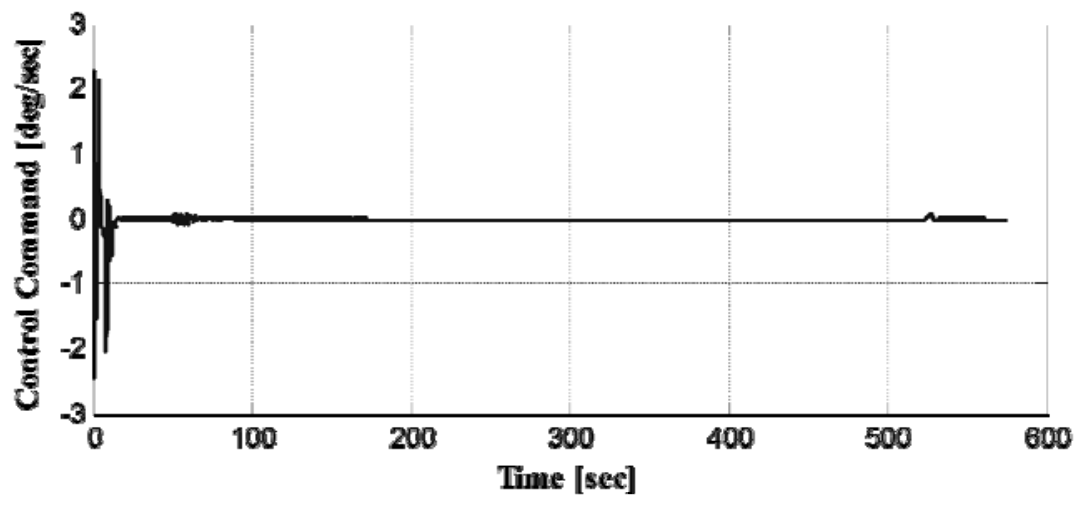

Fig. 9. Control deflection vs. time. 
Table 3. Trajectory Dispersion Sources.

\begin{tabular}{ll}
\hline \hline Error sources & Deviation \\
\hline Thrust misalignment & $0.25 \mathrm{deg}$ \\
Drag coefficient & $6.7 \%$ \\
Thrust variation & $2 \%$ \\
\hline \hline
\end{tabular}

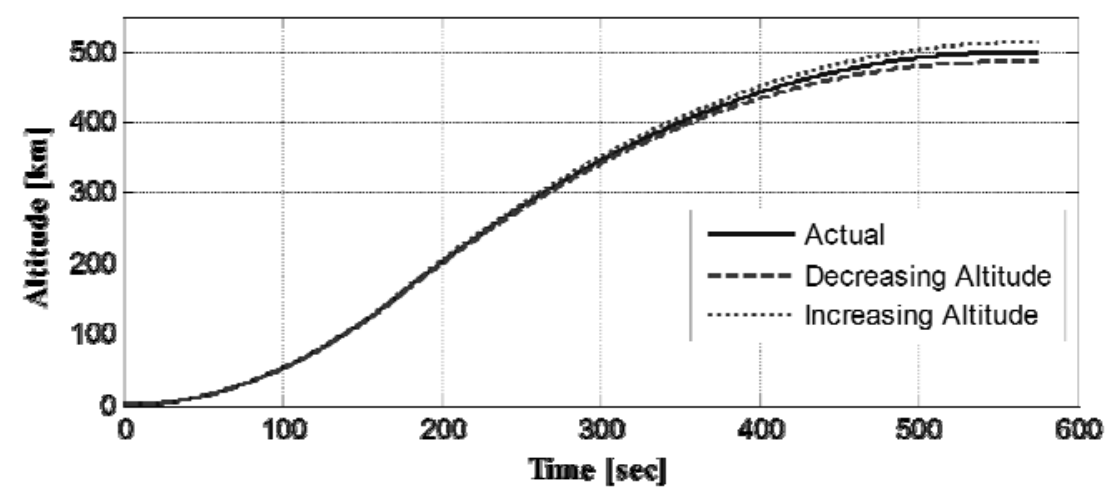

Fig. 10. Altitude with applied disturbances.

These disturbances change the pitching behavior which in turn increase or decrease the altitude. To cater the difference in the altitude, a guidance mechanism is applied which reshapes reference pitch angle online and manage to get the desired altitude. Results show in both cases that the performance of AFSMC is very robust and attitude is accurately followed even in perturbed conditions as shown in Fig. $11-16$.

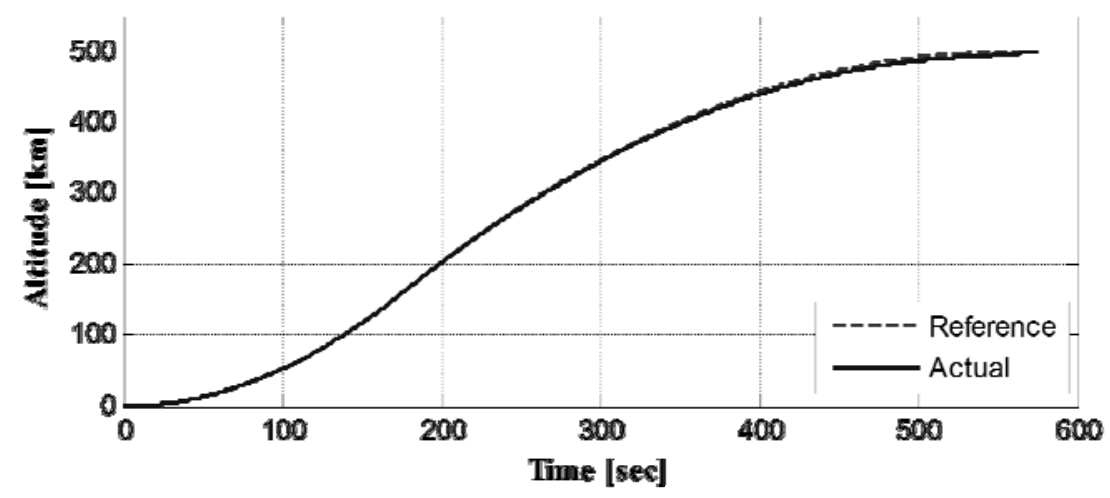

Fig. 11. Injection altitude with increasing altitude disturbance. 


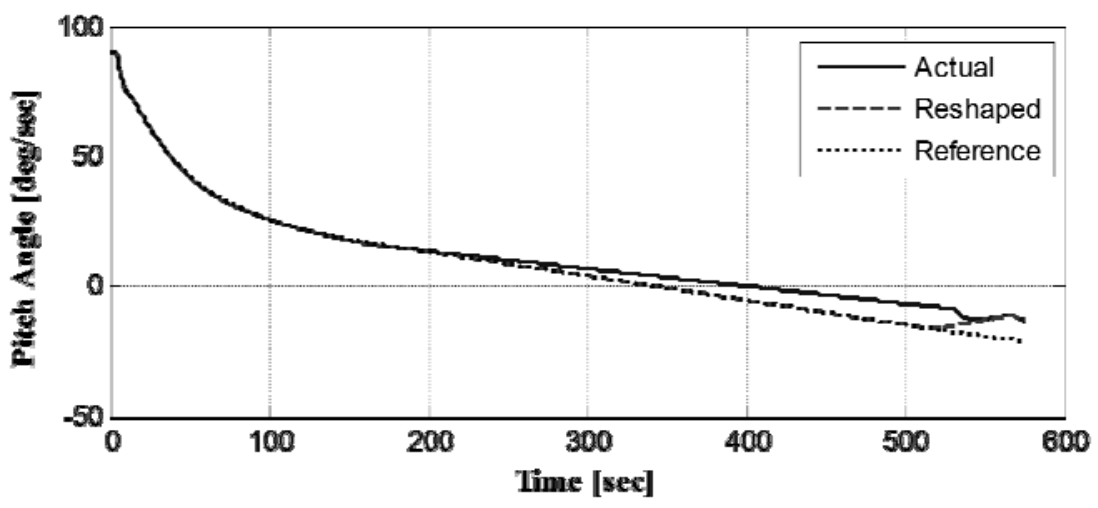

Fig. 12. Pitch control with Increasing Altitude disturbance.

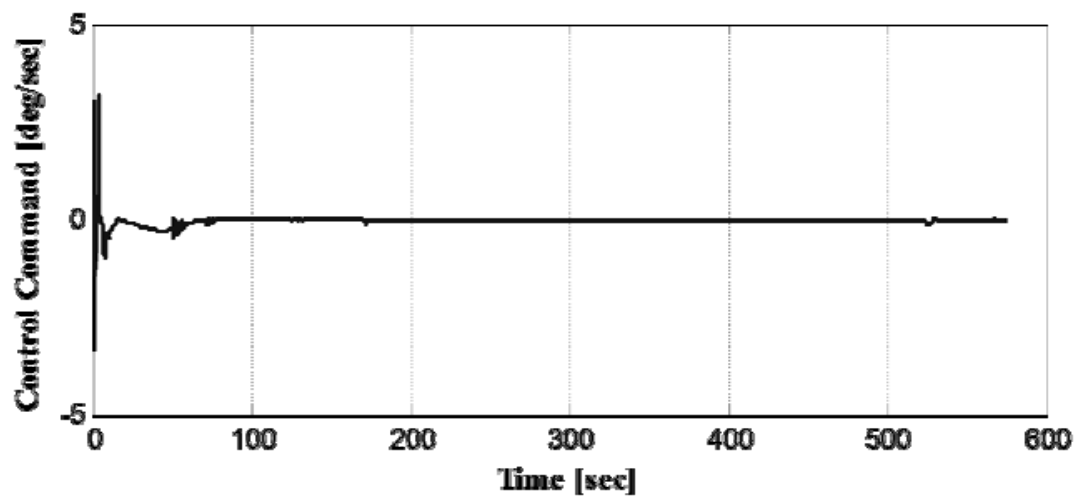

Fig. 13. Control deflection with Increasing altitude disturbance.

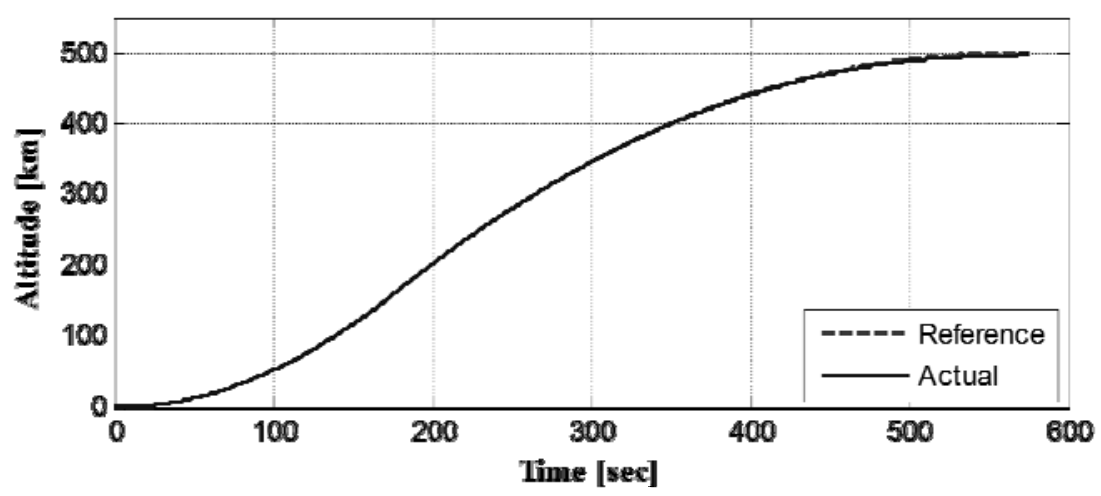

Fig. 14. Injection altitude with decreasing altitude disturbance. 


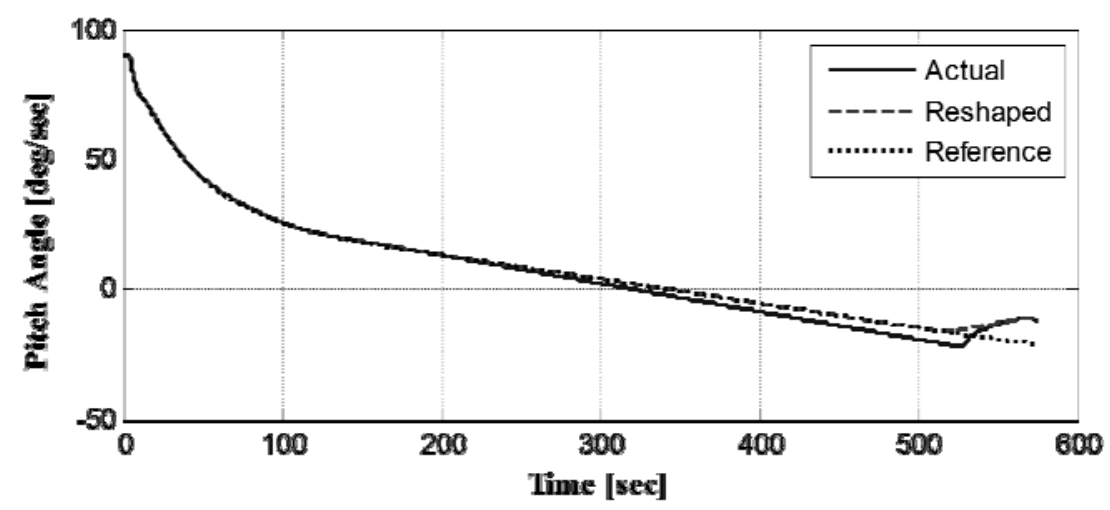

Fig. 15. Pitch control with decreasing Altitude disturbance.

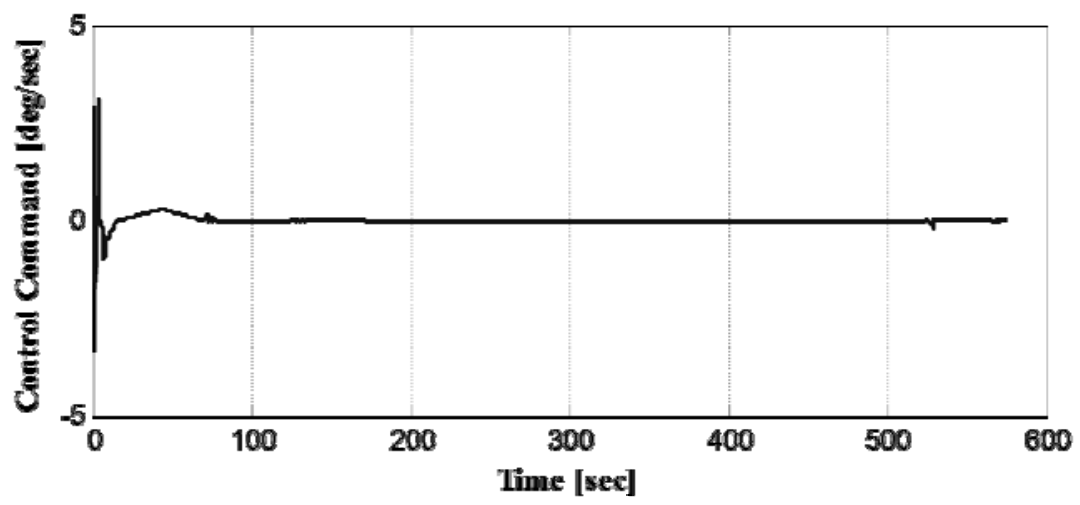

Fig. 16. Control deflection with decreasing altitude disturbance.

\section{Conclusion}

The paper presents the design of AFSMC methodology and its implementation on pitch attitude control of a SLV. In SIMULINK/MATLAB ${ }^{\circledR}$ environment, a $3 \mathrm{DOF}$ model is developed to simulate the ascent flight trajectory of the SLV. In AFSMC control, fuzzy gains are automatically adjusted by using Lyapunov stability such that an adaptation law is constructed to ensure closed loop system stability. Moreover, an estimation mechanism is designed in the switching control part of the SMC law to determine the bound of estimation error in real time. An external guidance loop is designed to increase injection accuracy of the SLV in perturbed flight conditions by reshaping the reference pitch attitude profile in real time. Results of 3DOF simulations demonstrate the efficacy of designed AFSMC, which accurately tracks the reshaped pitch attitude profile even in the presence of disturbances. 


\section{References}

[1] Mamdani, E. H., "Applications of fuzzy algorithms for simple dynamic plants", Proc. IEE 121: $1585-1588$ (1974).

[2] Lee, C. C., "Fuzzy logic in control systems: fuzzy logic controller-Part I/II", IEEE Transactions on Systems, Man, and Cybernetics, 20: 404-435 (1990).

[3] Abonyi, J., “Fuzzy Model Identification for Control”, Birkhauser Boston (2002).

[4] Kim, S. W. and Lee, J. J., "Design of a fuzzy controller with fuzzy sliding surface", Fuzzy Sets Systems, 71: 359-367 (1995).

[5] Yu, X., Man, Z. and Wu, B., "Design of fuzzy sliding-mode control systems", Fuzzy Sets and Systems, 95: 295-306 (1998).

[6] Palm, R., "Robust Control by Fuzzy Sliding Mode," Automatica, 30:1429-1437 (1994).

[7] Wang, L. X., "Adaptive Fuzzy Systems and Control: Design and Stability Analysis", Prentice-Hall, Englewood CliEs, NJ, (1994).

[8] Chekireb, H., Tadjine, M. and Bouchaffra, D., "Direct adaptive fuzzy control of nonlinear system class with application", Control and Intelligent Systems, 21(2): 113-121 (2002).

[9] Chen, Y. J., "Fuzzy sliding mode controller design: indirect adaptive approach", Cybernetic Systems, 30(1): 9-27 (1999).

[10] Roopaei, M., Zolghadri, M. and Meshksar, S., "Enhanced adaptive fuzzy sliding mode control for uncertain nonlinear systems", Communications in Nonlinear Science and Numerical Simulation, Elsevier, 14(9): 3670-3681 (2009).

[11] Saghafinia, A., Ping, H. W., Uddin, M. N. and Gaeid, K. S., "Adaptive Fuzzy SlidingMode Control Into Chattering-Free IM Drive", Industry Applications, IEEE Transactions, 51(1): 692-701 (2015).

[12] Li, Y., Wang,. H., Zhao, B. and Liu, K., "Adaptive Fuzzy Sliding Mode Control for the Probe Soft Landing on the Asteroids with Weak Gravitational Field", Mathematical Problems in Engineering, Hindawi Publishing Corporation (2015).

[13] Wang, Z., Wang, Q. and Zhang, X., “An Adaptive Fuzzy Sliding-mode Technique for Attitude Control of Launch Vehicle", International Conference on Mechatronics and Automation, ICMA, pp: 1587-1592 (2007).

[14] Ansari, U. and Alam, S., "Hybrid Genetic Algorithm Fuzzy Rule Based Guidance and Control of Satellite Launch Vehicle", ISDA 2011, Cordoba, Spain, 22-24 Nov (2011).

[15] Ansari, U., Alam, S. and Jafri, S. M., "Trajectory Optimization and Adaptive Fuzzy based Attitude Control of Satellite Launch Vehicle" MED 2012, Barcelona, Spain, 03-06 July (2012).

[16] Ansari, U. and Bajodah, A. H., "Generalized Dynamic Inversion Scheme for Satellite Launch Vehicle Attitude Control", 1st IFAC Workshop on Advanced Control and Navigation for Autonomous Aerospace Vehicles, Seville, Spain, June (2015).

[17] Wang, W., "Adaptive fuzzy sliding mode control for inverted pendulum", Proceedings of the Second Symposium International Computer Science And Computational Technology Huangshan, China, pp: 231-234 (2009). 
[18] Qazi, M. and Linshu, H., "Comparison of some Direct Search Methods for Conceptual Design of Multistage Space Launch Vehicle", IFSA World Congress, pp: 1364-1369, (2005).

[19] Xiao, Y., "Rocket Ballistics and Dynamics". Beijing University of Aeronautics and Astronautics, China (2007).

[20] Alam, S., Jafri, S. M. and Ansari, U., "Robust Integral Sliding Mode Control for NonLinear system", 2nd International Conference Aerospace Science and Engineering ICASE, Islamabad, Pakistan, 27-29 Dec (2011).

[21] Ansari, U., Alam, S. and Jafri, S. M., "Depth Control for an Autonomous Underwater Vehicle using Fuzzy Controller" 8th IBCAST Islamabad, Pakistan, 10-13 Jan. (2011).

[22] Wang, L. X., "Stable adaptive fuzzy control of nonlinear systems," IEEE Trans. Fuzzy Systems, 1: 146-155 (1993).

[23] Wang, L. X., "Stable adaptive fuzzy controllers with application to inverted pendulum tracking," IEEE Transactions on Systems, Man, and Cybernetics, 26(5): 677-690 (1996).

[24] Wang, Y. and Fei, J., "Adaptive fuzzy sliding mode control for PMSM position regulation system", International Journal of Innovative Computing, Information and Control, 11(3): 881-891 (2015).

[25] Khalil, H.K., "Nonlinear Systems", Prentice Hall; $3^{\text {rd }}$ edition, (2001). 
تطبيق لطريقة التحكم التكيفي بالنسق الانزلاقي التكاملي المدمج

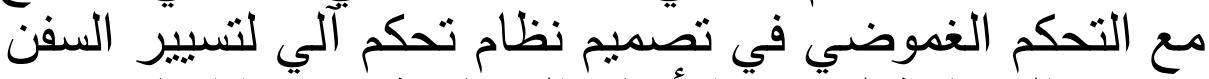
الفضائية لتشثين الأقمار الصناعية في مداراتها عزير أنصاري و عبد الرحمن حسن باجودة

قسم هندسة الطبيان، كلية الهندسة، جامعة الملك عبد العزيز، جدة، المدلكة العربية السعودية

المستخلص. تعرض الورقة العلمية نطبيقاً لطريقة النحكم الآلي النكيفي

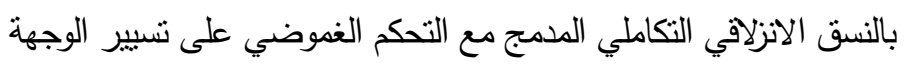
الطولية لسفينة فضائية لتشين الأقمار الصناعية بإيلاجها في مداراتها.

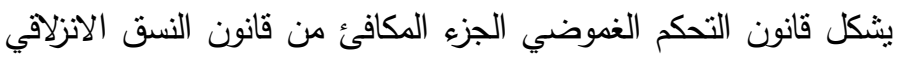

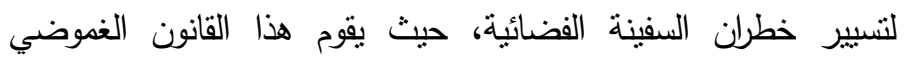

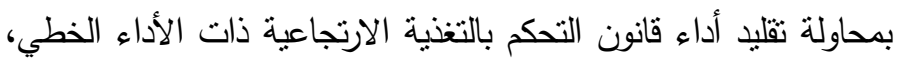

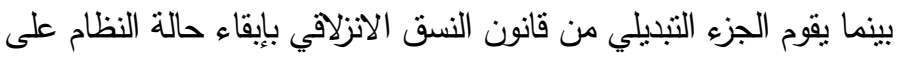

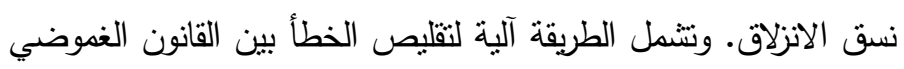

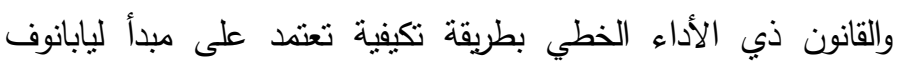
لضمان الاستقرار التقاربي لكتيزير الخطأ. كما يثمل التصميم دائرة توجيه

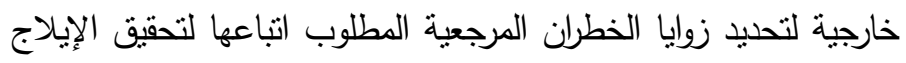

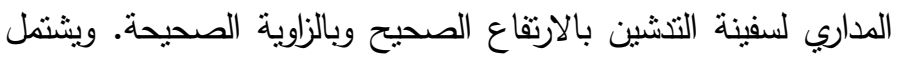

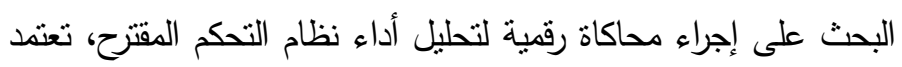

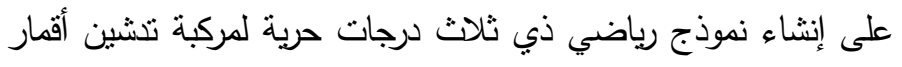

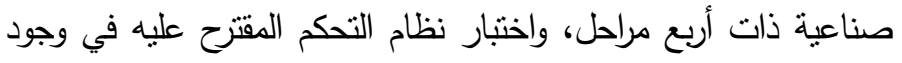
مؤثرات خارجية وداخلية ولايقين في النموذج الرياضي.

الكلمات الدفتاحية: التحكم في وجهة سفينة فضائية، التحكم التكيفي

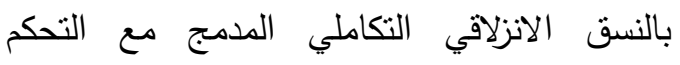
الغوضي، مبدأ ليابونوف للاستقرار، نوجيه سفينة فضائية، تقدير الزمن الحقيقي. 